\title{
Apparent prevention of neural tube defects by periconceptional vitamin supplementation
}

\author{
R W SMITHELLS, S SHEPPARD, C J SCHORAH, M J SELLER, N C NEVIN, R HARRIS, A P READ, \\ AND D W FIELDING
}

Department of Paediatrics and Child Health, University of Leeds; Paediatric Research Unit, Guy's Hospital, London; Department of Medical Genetics, Queen's University of Belfast; Department of Medical Genetics, University of Manchester; Department of Paediatrics, Chester

SUMmARY An eariier preliminary paper is expanded. Women who had given birth to one or more infants with a neural tube defect were recruited into a trial of periconceptional vitamin supplementation. Two hundred mothers attending five centres were fully supplemented (FS), 50 were partially supplemented (PS), and 300 were unsupplemented (US). Neural tube defect recurrences in the study pregnancies were $1(0.5 \%)$, in FS, none in PS, and $13(4 \%)$ in US mothers. The difference in outcome between FS and US mothers is significant. The most likely explanation is that supplementation has prevented some neural tube defects, but fu rther studies are needed.

It is nearly half a century since Hale ${ }^{1}$ demonstrated an association between nutritional deficiency in a pregnant mammal and malformation in the offspring. His pioneer experiments established a causal relationship between vitamin A deficiency and a variety of birth defects, notably anophthalmia, in pigs. Subsequently Warkany and Nelson ${ }^{2}$ showed that malformations could be produced in rodents by the manipulation of maternal diet.

There is as yet no clear evidence to link nutritional deficiency with malformations in the human. However, there is considerable circumstantial evidence pointing to such an association. The widely observed social class gradient in the incidence of neural tube defects (NTD) suggests the possibility of a nutritional contribution to their causation. Significant social class differences in nutrient intake ${ }^{3}$ and in blood levels of folic acid, ascorbic acid, and riboflavin ${ }^{4}$ in early pregnancy have been reported. An association between abnormal folate metabolism at the end of pregnancy and NTD has been suggested ${ }^{5}$ but not confirmed. ${ }^{6} \mathrm{~A}$ relationship between poor diet and NTD recurrence, with possible benefit of dietary counselling, has recently been reported. ${ }^{7}$

In a prospective study of blood vitamin levels in the first trimester of pregnancy, mean blood levels of folate, ascorbic acid, and riboflavin were lower in a small group of mothers who later gave birth to infants with central nervous system malformations than in controls, the differences being significant for red cell folate and leucocyte ascorbic acid. ${ }^{4}$ To test the hypothesis that minor deficiencies of one or more vitamins may contribute to the causation of NTD, an intervention study has been carried out. Following our preliminary paper ${ }^{8}$ we now report the results of further analysis and follow-up. The figures in this paper do not match precisely those in the earlier one. The main difference results from the inclusion here of partially supplemented mothers (defined below) and some additional unsupplemented mothers associated with them. Some minor adjustments result from more detailed scrutiny of our original data.

\section{Patients and methods}

This is a multicentre study. The participating centres and their related areas were: Leeds (Yorkshire region), Guy's Hospital, London (South-east England), Belfast (Northern Ireland), Manchester (North-west region), Chester (Cheshire).

We decided to offer periconceptional multivitamin supplementation to women who had already had at least one NTD infant,* and who therefore faced a risk of recurrence of about $5 \%$. Our original proposal for a double-blind placebo study was rejected by two research ethics committees

*For the purposes of the study, NTD includes anencephaly, encephalocele, cranial meningocele, iniencephaly, myelocele, myelomeningocele, and meningocele. It excludes isolated hydrocephalus and spina bifida occulta. 
(see below) and an alternative design was therefore adopted. This offered vitamin supplementation to all eligible women recruited to the study - that is, to any woman who had previously given birth to at least one NTD child, who was not pregnant, and who planned a further pregnancy at some time in the future. Such women were referred to the study from genetic counselling clinics, or by obstetricians, general practitioners, and health visitors. Supplementation was effected with Pregnavite Forte F (Bencard), 1 tablet three times a day, which provides daily vitamin A $4000 \mathrm{IU}$, vitamin D 400 IU, thiamine $1.5 \mathrm{mg}$, riboflavin $1.5 \mathrm{mg}$, pyridoxine $1 \mathrm{mg}$, nicotinamide $15 \mathrm{mg}$, ascorbic acid $40 \mathrm{mg}$, folic acid $0.36 \mathrm{mg}$, ferrous sulphate equivalent to $75.6 \mathrm{mg}$ $\mathrm{Fe}$, and calcium phosphate $480 \mathrm{mg}$. This was chosen as one of the few available preparations containing folic acid, ascorbic acid, and riboflavin.

Women planning an immediate pregnancy were started on vitamin supplementation at once. Those planning a pregnancy later were asked to let us know when they felt ready for another pregnancy but before they became pregnant. All were asked to take the tablets for not less than 28 days before conception, and to continue at least until the date of the second missed period. Those who complied were regarded as 'fully supplemented'. Those who conceived within 28 days of starting supplementation, or who started taking vitamins after conception (but before the estimated time of neural tube closure), or were known to have missed their tablets for more than 1 day, were regarded as 'partially supplemented'.* In Northern Ireland, Yorkshire, and Cheshire, women taking oral contraceptives were asked to adopt an alternative means of contraception from the date of starting vitamins because oral contraceptives lower blood levels of certain vitamins. ${ }^{9}$

The duration of preconceptional supplementation varied widely, the mean being 110 days. A few women took many months -in 7 cases more than a year-to conceive. In view of the remote possibility that vitamin A might be teratogenic in humans, ${ }^{10}$ serum vitamin A levels were checked in 2 of these women after 12 months of continuous supplementation and were found to be within the normal range.

After the second month of pregnancy, Pregnavite Forte $F$ was stopped. Any subsequent iron and vitamin supplementation was at the discretion of the

*The number of days of preconceptional supplementation was calculated from the date of the last menstrual period and the length of the menstrual cycle, the estimated date of conception being 14 days before the first missed period. If periods were irregular the calculation was based on a 28-day cycle. general practitioner and obstetrician. As all supplemented women had had at least one previous NTD infant, all were offered amniocentesis, and most accepted.

For comparative purposes, in the absence of a true control group, it was felt important to have information about NTD recurrences in the offspring of unsupplemented mothers drawn from the same places and over the same period of time as the supplemented mothers. The unsupplemented group comprised women who had also had at least one NTD infant and who were already pregnant when referred but not beyond the first trimester (except in Manchester).

We thought that the social class distribution of the two groups might differ. Furthermore, as supplemented mothers were recruited before con- $\vec{N}$ ception and most unsupplemented mothers a variable time after, information regarding events in early pregnancy is not strictly comparable.

\section{Results}

In Tables 1-9 mothers and their infants or fetuses are classified as fully supplemented (FS), partially $\stackrel{\mathbb{Q}}{-}$ supplemented (PS), or unsupplemented (US). Of $50 \overrightarrow{0}$ PS mothers, 34 had some preconceptional (mo⿺𠃊丶 than 10 days in 25) and full postconception supplementation: 16 started taking supplements 0-25 days after conception. In Tables 2-9 figures in parentheses indicate the number of NTD recurrences in study pregnancies.

Because the women studied were not randomly allocated to supplemented or unsupplemented $\overrightarrow{\overrightarrow{0}}$ groups, it is important to compare the groups in 3 respect of factors which might influence the risk of NTD recurrence. Comparisons are made in relation to referral centre, number of previous NTDs, social class, maternal age, and outcome of ? previous pregnancy.

Referral centre. The reported incidence and re- $\frac{9}{3}$ currence rate of NTDs are higher in Northern $\mathrm{O}$ Ireland than in South-east England, the rates for northern England being intermediate. Table 1 lists $\frac{D}{0}$ the number of mothers recruited for supplementation in each centre, the number of withdrawals and $N$ exclusions (with reasons), the number fully and $N$ partially supplemented, and the number unsupple- $N$ mented. Of 74 not yet conceived, 18 withdrew from $\omega$ the study before conception. The remaining 56, many of whom were recruited in the last few months of the first cohort, remain in the study as part of a second cohort. Of 15 with erratic supplementation, 13 withdrew, mainly because of alleged side effects 
Table 1 Numbers of supplemented and unsupplemented mothers, by centre, with reasons for withdrawals and exclusions

\begin{tabular}{|c|c|c|c|c|c|}
\hline \multirow[t]{2}{*}{ Mothers } & \multicolumn{5}{|l|}{ Centre } \\
\hline & Leeds & $\begin{array}{l}\text { London } \\
\text { (Guys) }\end{array}$ & Belfast & $\begin{array}{l}\text { Man- } \\
\text { chester }\end{array}$ & Chester \\
\hline $\begin{array}{l}\text { Recruited for supplement- } \\
\text { ation }(\mathrm{n}=342)\end{array}$ & 56 & 132 & 92 & 47 & 15 \\
\hline $\begin{array}{l}\text { Withdrawals and exclusions } \\
\text { (see below) }(n=92)\end{array}$ & & & & & \\
\hline $\begin{array}{l}\text { (see below) }(\mathrm{n}=92) \\
\text { Supplemented }\end{array}$ & 10 & 38 & 30 & 8 & 6 \\
\hline Total $(n=250)$ & 46 & 94 & 62 & 39 & 9 \\
\hline Fully $(n=200)^{*}$ & 38 & 70 & 53 & 31 & 8 \\
\hline Partially $(n=50) \dagger$ & 8 & 24 & 9 & 8 & 1 \\
\hline Withdrawals and exclusions & & & & & \\
\hline $\begin{array}{l}\text { Not yet conceived }(n=74) \\
\text { Supplementation erratic }\end{array}$ & 8 & 31 & 24 & 5 & 6 \\
\hline$(n=15)$ & 2 & 6 & 5 & 2 & $\mathbf{0}$ \\
\hline Moved from area $(n=3)$ & 0 & 1 & 1 & 1 & 0 \\
\hline $\begin{array}{l}\text { Unsupplemented } \\
(\mathbf{n}=301) \ddagger^{*}\end{array}$ & 35 & 95 & $125 \ddagger$ & 36 & 10 \\
\hline
\end{tabular}

Table 2 Numbers of mothers and infants or fetuses by centre and number of previous NTDs, with NTD recurrences (in parentheses)

\begin{tabular}{|c|c|c|c|c|c|c|}
\hline \multirow[t]{2}{*}{ Centre } & \multicolumn{3}{|c|}{ One previous NTD } & \multicolumn{3}{|c|}{ Two previous NTDs } \\
\hline & $F S$ & $P S$ & $U S$ & $F S$ & $P S$ & US \\
\hline $\begin{array}{l}\text { Leeds } \\
\text { Guy's } \\
\text { Belfast } \\
\text { Manchester } \\
\text { Chester } \\
\text { All mothers } \\
\text { Twin pairs }\end{array}$ & $\begin{array}{c}36 \\
62(1) \\
47 \\
29 \\
8 \\
182(1) \\
4\end{array}$ & $\begin{array}{r}8 \\
22 \\
9 \\
7 \\
1 \\
47 \\
2\end{array}$ & $\begin{array}{c}32(1) \\
87(3) \\
108(5) \\
33 \\
10 \\
270(9) \\
4\end{array}$ & $\begin{array}{r}2 \\
8 \\
6 \\
2 \\
0 \\
18 \\
0\end{array}$ & $\begin{array}{l}0 \\
2 \\
0 \\
1 \\
0 \\
3 \\
0\end{array}$ & $\begin{array}{c}3(1) \\
8(2) \\
16(1) \\
3 \\
0 \\
30(4) \\
1\end{array}$ \\
\hline $\begin{array}{l}\text { All infants or fetuses } \\
\text { Spontaneous abor- } \\
\text { tions not examined } \\
\text { Infants or fetuses } \\
\text { examined }\end{array}$ & $\begin{array}{l}186(1) \\
\text { d } 9 \\
177(1)\end{array}$ & $\begin{array}{r}49 \\
1\end{array}$ & $\begin{array}{c}10 \\
264(9)\end{array}$ & 18 & 3 & $\begin{array}{c}31(4) \\
0 \\
31(4)\end{array}$ \\
\hline
\end{tabular}

from the tablets. We do not know if any was pregnant at the time of withdrawal. The other 2 were excluded because of gross unreliability: one of them subsequently had a healthy baby, the other made arrangements for termination of an unwanted pregnancy.

The proportion of supplemented to unsupplemented mothers in each centre is broadly comparable except for Belfast where there was an excess of unsupplemented.

Number of previous NTDs (Table 2). The recurrence risk is known to be higher after 2 previous NTDs than after one. Among unsupplemented mothers, 270 had each had one previous NTD infant of whom $9(3 \%)$ had recurrences in the study pregnancy, compared with $4(13 \%)$ of 30 among those who had had 2 previous NTDs. In subsequent tables mothers with 1 and 2 previous NTDs are treated separately. The proportions of FS and US mothers with 2 previous NTDs are comparable $(9 \%$ and $10 \%$ respectively).

Table 2 also gives the number of twin pairs, the number of unexamined spontaneous abortions, and hence the number of examined infants or fetuses for which presence or absence of NTD is known.

Maternal age (Table 3). There is no consistent reported effect of maternal age on NTD incidence or recurrence, although the incidence has been higher among younger mothers in some studies, and among older mothers in others. The highest NTD recurrence rate in unsupplemented mothers was for the quinquennium $25-29$ years, $9(8 \%)$ of 116. The rate for mothers under 25 was $2(2 \%)$ of 94 , and for mothers 30 or more $2(2 \%)$ of 90 . The proportion of mothers aged 25-29 years was higher among the supplemented than the unsupplemented (FS $47 \%$, PS $40 \%$, US $39 \%$ ) so the slight difference

Table 3 Age distribution of mothers fully supplemented (FS), partially supplemented (PS), and unsupplemented (US) by centre

\begin{tabular}{|c|c|c|c|c|c|c|c|c|c|c|c|c|c|c|c|c|c|c|c|c|c|c|c|c|}
\hline \multirow[t]{2}{*}{ Centre } & \multicolumn{3}{|c|}{ 15-19 years } & \multicolumn{3}{|c|}{$20-24$ years } & \multicolumn{3}{|c|}{ 25-29 years } & \multicolumn{3}{|c|}{ 30-34 years } & \multicolumn{3}{|c|}{ 35-39 years } & \multicolumn{3}{|c|}{ 40-44 years } & \multicolumn{3}{|c|}{ Age not known } & \multicolumn{3}{|l|}{ Total } \\
\hline & $F S$ & $P S$ & US & $F S$ & $P S$ & $U S$ & $F S$ & $P S$ & $U S$ & $F S$ & $P S$ & US & $F S$ & $P S$ & $U S$ & $F S$ & $P S$ & $U S$ & $F S$ & $P S$ & US & $F S$ & $P S$ & $S U S$ \\
\hline $\begin{array}{l}\text { Leeds } \\
\text { Guy's } \\
\text { Belfast } \\
\text { Manchester } \\
\text { Chester }\end{array}$ & $\begin{array}{l}\mathbf{0} \\
\mathbf{0} \\
\mathbf{0} \\
\mathbf{1} \\
\mathbf{0}\end{array}$ & $\begin{array}{l}0 \\
1 \\
1 \\
1 \\
0\end{array}$ & $\begin{array}{l}1 \\
1 \\
1 \\
1 \\
0\end{array}$ & $\begin{array}{l}8 \\
13(1) \\
16 \\
9 \\
2\end{array}$ & $\begin{array}{l}4 \\
8 \\
2 \\
2 \\
0\end{array}$ & $\begin{array}{l}12 \\
32(2) \\
28 \\
15 \\
3\end{array}$ & $\begin{array}{r}21 \\
32 \\
23 \\
14 \\
4\end{array}$ & $\begin{array}{l}4 \\
7 \\
4 \\
4 \\
1\end{array}$ & $\begin{array}{c}17(2) \\
42(3) \\
39(4) \\
14 \\
4\end{array}$ & $\begin{array}{r}9 \\
21 \\
13 \\
6 \\
2\end{array}$ & $\begin{array}{l}0 \\
7 \\
2 \\
1 \\
0\end{array}$ & $\begin{array}{l}5 \\
17 \\
41(1) \\
5 \\
3\end{array}$ & $\begin{array}{l}\mathbf{0} \\
\mathbf{3} \\
\mathbf{1} \\
\mathbf{0} \\
\mathbf{0}\end{array}$ & $\begin{array}{l}0 \\
1 \\
0 \\
0 \\
0\end{array}$ & $\begin{array}{r}0 \\
3 \\
11 \\
1 \\
0\end{array}$ & $\begin{array}{l}\mathbf{0} \\
\mathbf{0} \\
\mathbf{0} \\
1 \\
0\end{array}$ & $\begin{array}{l}\mathbf{0} \\
\mathbf{0} \\
\mathbf{0} \\
\mathbf{0} \\
\mathbf{0}\end{array}$ & $\begin{array}{l}0 \\
0 \\
4(1) \\
0 \\
0\end{array}$ & $\begin{array}{l}\mathbf{0} \\
\mathbf{1} \\
\mathbf{0} \\
\mathbf{0} \\
\mathbf{0}\end{array}$ & $\begin{array}{l}0 \\
0 \\
0 \\
0 \\
0\end{array}$ & $\begin{array}{l}0 \\
0 \\
0 \\
0 \\
0\end{array}$ & $\begin{array}{l}38 \\
70(1) \\
53 \\
31 \\
8\end{array}$ & $\begin{array}{r}8 \\
24 \\
9 \\
8 \\
1\end{array}$ & $\begin{array}{c}35(2) \\
95(5) \\
124(6) \\
36 \\
10\end{array}$ \\
\hline $\begin{array}{l}\text { All } \\
\%\end{array}$ & $\begin{array}{l}1 \\
0.5\end{array}$ & $\begin{array}{l}3 \\
6\end{array}$ & $\begin{array}{l}4 \\
1\end{array}$ & $\begin{array}{l}48(1) \\
24\end{array}$ & $\begin{array}{l}16 \\
32\end{array}$ & $\begin{array}{l}90(2) \\
30\end{array}$ & $\begin{array}{l}94 \\
47\end{array}$ & $\begin{array}{l}20 \\
40\end{array}$ & $\begin{array}{l}116(9) \\
39\end{array}$ & $\begin{array}{l}51 \\
26\end{array}$ & $\begin{array}{l}10 \\
20\end{array}$ & $\begin{array}{l}71(1) \\
24\end{array}$ & $\begin{array}{l}4 \\
2\end{array}$ & $\begin{array}{l}1 \\
2\end{array}$ & $\begin{array}{r}15 \\
5\end{array}$ & $\begin{array}{l}1 \\
0.5\end{array}$ & $\begin{array}{l}0 \\
0\end{array}$ & $\begin{array}{l}4(1) \\
1\end{array}$ & $\begin{array}{l}1 \\
0.5\end{array}$ & $\begin{array}{l}0 \\
0\end{array}$ & $\begin{array}{l}0 \\
0\end{array}$ & $\begin{array}{l}200(1) \\
100\end{array}$ & $\begin{array}{r}50 \\
100\end{array}$ & $\begin{array}{l}300(13) \\
100\end{array}$ \\
\hline$\%$ & $\begin{array}{l}4 \\
2\end{array}$ & & & $\begin{array}{l}64( \\
26\end{array}$ & & & & $\begin{array}{l}14 \\
46\end{array}$ & & 2 & & & 2 & & & & .4 & & & & & & & \\
\hline
\end{tabular}

Figures in parentheses are number of NTDs. 


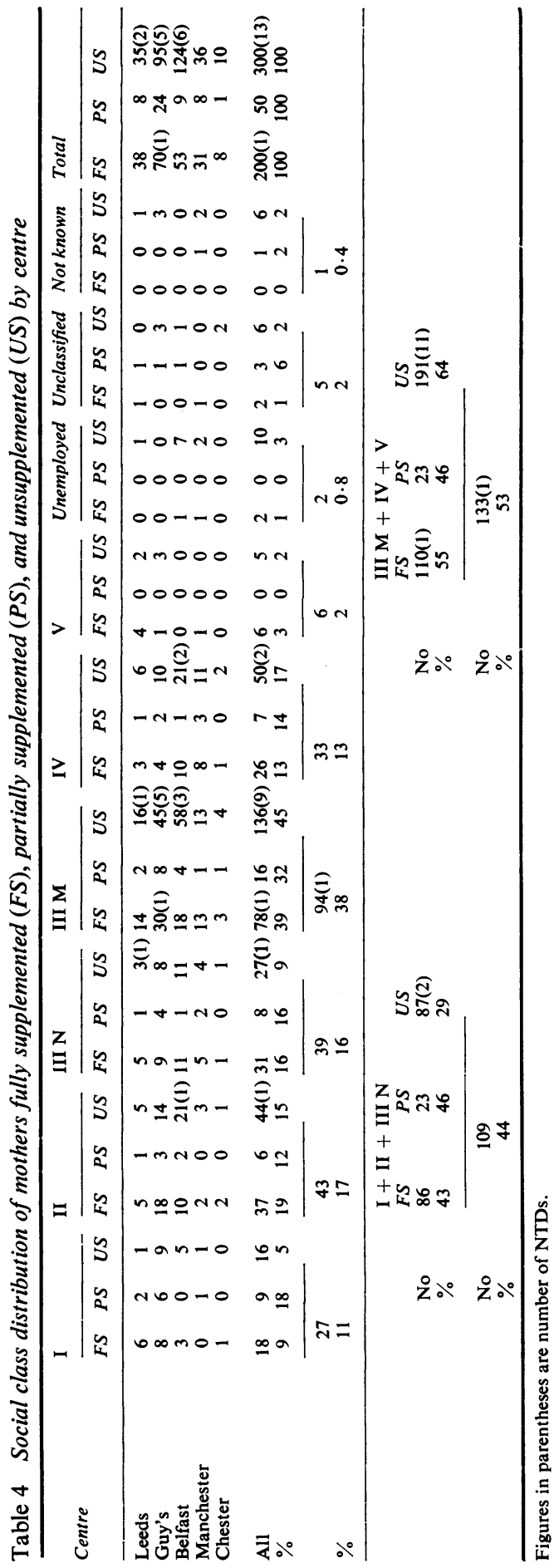


in age distribution would tend to diminish any preventive effect of supplementation. In subsequent analyses therefore, maternal age is ignored.

Social class (Table 4). A rising NTD incidence from class $I$ to $V$ is well known, and data from Belfast ${ }^{11}$ suggest a comparable gradient in recurrence rate. Among unsupplemented women there was $1(2 \%)$ recurrence in 60 class $I$ and II mothers, $1(4 \%)$ in 27 class III non-manual, $9(7 \%)$ in 136 class III manual, and $2(7 \%)$ recurrences in 55 class IV and V. If class III non-manual is grouped with I and II, and III manual with classes IV and $\mathrm{V}$, the respective recurrence rates are $2 \%$ and $6 \%$. The proportion of mothers in classes I + II + IIIN was higher among the supplemented than among the unsupplemented (FS $43 \%$, PS $46 \%$, US $29 \%$ ) and for classes IIIM + IV + V the reverse (FS $55 \%$, PS $46 \%$,
US $64 \%$ ). In subsequent analyses these two broad social groupings are separated.

Previous reproductive history (Table 5). This is considered because of the known association between NTDs and preceding spontaneous abortions. ${ }^{12}$ The overall previous reproductive performances of FS, PS, and US mothers were broadly comparable with regard to numbers of previous pregnancies, NTDs, and spontaneous abortions (Table 5). Considerably more unsupplemented than supplemented study pregnancies were immediately preceded by a spontaneous abortion (17\% US compared with $8 \%$ FS). The relevance of this observation is discussed later.

Outcome of pregnancies (Tables 6 and 7). The outcomes of pregnancies of FS mothers are shown in

Table 5 Previous reproductive history

\begin{tabular}{|c|c|c|c|c|c|c|}
\hline & \multicolumn{3}{|c|}{ One previous $N T D$} & \multicolumn{3}{|c|}{ Two previous NTDs } \\
\hline & $\begin{array}{l}F S \\
(n=182)\end{array}$ & $\begin{array}{l}P S \\
(n=47)\end{array}$ & $\begin{array}{l}U S \\
(n=270)\end{array}$ & $\begin{array}{l}F S \\
(n=18)\end{array}$ & $\begin{array}{l}P S \\
(n=3)\end{array}$ & $\begin{array}{l}U S \\
(n=30)\end{array}$ \\
\hline $\begin{array}{l}\text { Total (mean) no of previous pregnancies } \\
\text { Total (\%) previous spontaneous abortions } \\
\text { Outcome of pregnancy immediately before } \\
\text { study pregnancy }\end{array}$ & $\begin{array}{c}379(2 \cdot 1) \\
75(20)\end{array}$ & $\begin{array}{l}97(2 \cdot 1) \\
10(10)\end{array}$ & $\begin{array}{l}650(2 \cdot 4) \\
127(20)\end{array}$ & $\begin{array}{l}59(3 \cdot 3) \\
10(17)\end{array}$ & $\begin{array}{l}9(3 \cdot 0) \\
2(22)\end{array}$ & $\begin{array}{l}102(3 \cdot 4) \\
20(20)\end{array}$ \\
\hline $\begin{array}{l}\text { Spontaneous abortion (\%) } \\
\text { NTD (\%) }\end{array}$ & $\begin{array}{c}15(8) \\
144(79)\end{array}$ & $\begin{array}{c}3(6) \\
29(62)\end{array}$ & $\begin{array}{r}46 *(17) \\
161 *(60)\end{array}$ & $\begin{array}{r}2(11) \\
15(83)\end{array}$ & $\begin{array}{l}0 \\
2(67)\end{array}$ & $\begin{array}{r}5(17) \\
18(60)\end{array}$ \\
\hline
\end{tabular}

* Figures include 3 NTDs spontaneously aborted.

Table 6 Outcome of pregnancy in fully supplemented mothers with one previous NTD infant $(n=182:$ 4 twin pairs) by centre and social class

\begin{tabular}{|c|c|c|c|c|c|c|c|c|c|c|c|c|c|c|c|c|c|c|c|c|c|c|c|c|c|}
\hline \multirow[t]{3}{*}{ Social class } & \multirow{2}{*}{\multicolumn{6}{|c|}{ Live and stillbirths }} & \multicolumn{12}{|c|}{ Spontaneous abortions } & \multirow{2}{*}{\multicolumn{6}{|c|}{ Therapeutic abortions }} & \multirow[t]{3}{*}{ Total } \\
\hline & & & & & & & \multicolumn{6}{|c|}{ Examined } & \multicolumn{6}{|c|}{ Not examined } & & & & & & & \\
\hline & $L$ & $\boldsymbol{G}$ & $\boldsymbol{B}$ & $M$ & $C$ & Total & $L$ & $\boldsymbol{G}$ & $\boldsymbol{B}$ & $M$ & $\boldsymbol{C}$ & Total & $L$ & $\boldsymbol{G}$ & $\boldsymbol{B}$ & $\boldsymbol{M}$ & $C$ & Total & $\boldsymbol{L}$ & $\boldsymbol{G}$ & $\boldsymbol{B}$ & $M$ & $C$ & Total & \\
\hline $\begin{array}{l}\text { I + II } \\
\text { III N } \\
\text { III } \mathbf{M} \\
\text { IV + V } \\
\text { Unclassified } \\
\text { All }\end{array}$ & $\begin{array}{r}10 \\
4 \\
12 \\
5 \\
1 \\
32\end{array}$ & $\begin{array}{r}19 \\
8 \\
24 \\
4 \\
0 \\
55\end{array}$ & $\begin{array}{r}11 \\
9 \\
13 \\
7 \\
1 \\
41\end{array}$ & $\begin{array}{r}2 \\
5 \\
10 \\
9 \\
2 \\
28\end{array}$ & $\begin{array}{l}3 \\
1 \\
4 \\
1 \\
0 \\
9\end{array}$ & $\begin{array}{r}45 \\
27 \\
63 \\
26 \\
4 \\
165\end{array}$ & $\begin{array}{l}1 \\
1 \\
1 \\
0 \\
0 \\
3\end{array}$ & $\begin{array}{l}\mathbf{0} \\
\mathbf{0} \\
\mathbf{0} \\
\mathbf{0} \\
\mathbf{0} \\
\mathbf{0}\end{array}$ & $\begin{array}{l}1 \\
2 \\
2 \\
1 \\
0 \\
6\end{array}$ & $\begin{array}{l}\mathbf{0} \\
\mathbf{0} \\
1 \\
0 \\
0 \\
1\end{array}$ & $\begin{array}{l}\mathbf{0} \\
\mathbf{0} \\
\mathbf{0} \\
\mathbf{0} \\
\mathbf{0} \\
\mathbf{0}\end{array}$ & $\begin{array}{r}2 \\
3 \\
4 \\
1 \\
0 \\
10\end{array}$ & $\begin{array}{l}2 \\
0 \\
0 \\
1 \\
0 \\
3\end{array}$ & $\begin{array}{l}4 \\
0 \\
1 \\
0 \\
0 \\
5\end{array}$ & $\begin{array}{l}\mathbf{0} \\
\mathbf{0} \\
\mathbf{0} \\
\mathbf{0} \\
\mathbf{0} \\
\mathbf{0}\end{array}$ & $\begin{array}{l}0 \\
0 \\
0 \\
1 \\
0 \\
1\end{array}$ & $\begin{array}{l}\mathbf{0} \\
\mathbf{0} \\
\mathbf{0} \\
\mathbf{0} \\
\mathbf{0} \\
\mathbf{0}\end{array}$ & $\begin{array}{l}6 \\
0 \\
1 \\
2 \\
0 \\
9\end{array}$ & $\begin{array}{l}\mathbf{0} \\
\mathbf{0} \\
\mathbf{0} \\
\mathbf{0} \\
\mathbf{0} \\
\mathbf{0}\end{array}$ & $\begin{array}{l}1^{*} \\
0 \\
1(1) \\
0 \\
0 \\
2(1)\end{array}$ & $\begin{array}{l}\mathbf{0} \\
\mathbf{0} \\
\mathbf{0} \\
\mathbf{0} \\
\mathbf{0} \\
\mathbf{0}\end{array}$ & $\begin{array}{l}\mathbf{0} \\
\mathbf{0} \\
\mathbf{0} \\
\mathbf{0} \\
\mathbf{0} \\
\mathbf{0}\end{array}$ & $\begin{array}{l}\mathbf{0} \\
\mathbf{0} \\
\mathbf{0} \\
\mathbf{0} \\
\mathbf{0} \\
\mathbf{0}\end{array}$ & $\begin{array}{l}1 \\
0 \\
1(1) \\
0 \\
0 \\
2(1)\end{array}$ & $\begin{array}{c}54^{*} \\
30 \\
69(1) \\
29 \\
4 \\
186(1)\end{array}$ \\
\hline
\end{tabular}

Figures in parentheses are number of NTDs.

* Intrauterine death: normal fetus.

$\mathbf{L}=$ Leeds, $\mathbf{G}=$ Guy's, $\mathbf{B}=$ Belfast, $\mathbf{M}=$ Manchester, $\mathbf{C}=$ Chester.

Table 7 Outcome of pregnancy in fully supplemented mothers with two previous NTD infants $(n=18)$ by centre and social class

\begin{tabular}{|c|c|c|c|c|c|c|c|c|c|c|c|c|c|c|c|}
\hline \multirow[t]{3}{*}{ Social class } & \multirow{2}{*}{\multicolumn{6}{|c|}{ Live and stillbirths }} & \multicolumn{7}{|c|}{ Spontaneous abortions } & \multirow{3}{*}{$\begin{array}{l}\text { Therapeutic } \\
\text { abortions } \\
\text { All centres }\end{array}$} & \multirow[t]{3}{*}{ Total } \\
\hline & & & & & & & \multicolumn{6}{|c|}{ Examined } & \multirow{2}{*}{$\begin{array}{l}\text { Not examined } \\
\text { All centres }\end{array}$} & & \\
\hline & $L$ & $G$ & $B$ & $\boldsymbol{M}$ & $C$ & Total & $L$ & $\boldsymbol{G}$ & $\boldsymbol{B}$ & $M$ & $C$ & Total & & & \\
\hline $\begin{array}{l}\text { I }+ \text { II } \\
\text { III } \mathbf{N} \\
\text { III } \mathbf{M} \\
\text { IV + V } \\
\text { All }\end{array}$ & $\begin{array}{l}0 \\
0 \\
1 \\
1 \\
2\end{array}$ & $\begin{array}{l}2 \\
1 \\
4 \\
1 \\
8\end{array}$ & $\begin{array}{l}1 \\
0 \\
3 \\
2 \\
6\end{array}$ & $\begin{array}{l}0 \\
0 \\
1 \\
0 \\
1\end{array}$ & $\begin{array}{l}0 \\
0 \\
0 \\
0 \\
0\end{array}$ & $\begin{array}{r}3 \\
1 \\
9 \\
4 \\
17\end{array}$ & $\begin{array}{l}\mathbf{0} \\
\mathbf{0} \\
\mathbf{0} \\
\mathbf{0} \\
\mathbf{0}\end{array}$ & $\begin{array}{l}0 \\
0 \\
0 \\
0 \\
0\end{array}$ & $\begin{array}{l}\mathbf{0} \\
\mathbf{0} \\
\mathbf{0} \\
\mathbf{0} \\
\mathbf{0}\end{array}$ & $\begin{array}{l}0 \\
0 \\
1 \\
0 \\
1\end{array}$ & $\begin{array}{l}0 \\
0 \\
0 \\
0 \\
0\end{array}$ & $\begin{array}{l}0 \\
0 \\
1 \\
0 \\
1\end{array}$ & $\begin{array}{l}0 \\
0 \\
0 \\
0 \\
0\end{array}$ & $\begin{array}{l}0 \\
0 \\
0 \\
0 \\
0\end{array}$ & $\begin{array}{r}3 \\
1 \\
10 \\
4 \\
18\end{array}$ \\
\hline
\end{tabular}


Tables 6 and 7, and of US mothers in Tables 8 and 9, according to number of previous NTDs, referral centre, and social class. Spontaneous abortions are separated into those for which the abortus was examined and those for which this was not possible. Among the offspring of US mothers there were 13 (4\%) NTDs in 305 infants/fetuses. Of these, 9 (3\%) were born to 274 mothers with 1 previous NTD infant and $4(13 \%)$ to 31 mothers with 2 previous NTD infants. Comparable figures for FS mothers are $1(0.5 \%)$ NTD in 204 infants/fetuses overall, or $1(0.5 \%)$ in 186 born to mothers with 1 previous NTD. There were no recurrences in 18 born to mothers with 2 previous NTD infants.

For PS mothers (not shown in the tables) there were no recurrences in 49 offspring of mothers with 1 previous NTD infant or in 3 born to mothers with 2 previous NTD infants. For all supplemented mothers (FS + PS) there was 1 NTD among 256 infants/fetuses, a recurrence rate of $0.4 \%$.

If spontaneous abortions not examined are excluded from the calculations, the recurrence rates for US infants were $3 \%$ after 1 NTD, $13 \%$ after 2 NTDs, 4\% overall; for FS infants recurrence rates were $0.6 \%, 0.0 \%$, and $0.5 \%$. The lower recurrence rate in the FS compared with the US group

is significant $(\mathbf{P}<0.01$ one-tailed) by Fisher's exact method.

In view of the theoretical possibility that vitamin supplementation, whether or not preventing NTDs, might harm the embryo/fetus, an attempt was made to examine all babies of FS mothers for birth defects. In addition, as many as possible were later tested for developmental progress, hearing, and vision. Follow-up data are not available for the children of mothers who attended Guy's Hospital. For the other centres, significant birth defects recorded and results of examination at age 5 months to $3 \frac{1}{2}$ years are shown in Table 10.

Table 10 Results of examination

\begin{tabular}{lll}
\hline Centre & Birth defects & Follow-up \\
\hline Leeds $(\mathrm{n}=34)$ & $\begin{array}{c}1 \text { congenital heart } \\
\text { disease }\end{array}$ & $\begin{array}{c}28 \text { examined. Slight } \\
\text { developmental delay } \\
\text { in one }\end{array}$ \\
$\begin{array}{l}\text { Guy's }(\mathrm{n}=63) \\
\text { Belfast }(\mathrm{n}=47)\end{array}$ & $\begin{array}{l}1 \text { neural tube defect } \\
1 \text { jejunal atresia } \\
1 \text { mucopoly- } \\
\text { saccharidosis }\end{array}$ & $\begin{array}{c}22 \text { examined and } \\
\text { normal }\end{array}$ \\
Manchester $(\mathrm{n}=29)$ & $\begin{array}{c}\text { 26 examined and } \\
\text { normal. (3 sacral } \\
\text { dimples })\end{array}$ \\
Chester $(\mathrm{n}=9)$ & $\begin{array}{c}\text { examined and } \\
\text { normal }\end{array}$ \\
\hline
\end{tabular}

Table 8 Outcome of unsupplemented pregnancies in mothers with one previous NTD infant $(n=270:$ 4 twin pairs) by centre and social class

\begin{tabular}{|c|c|c|c|c|c|c|c|c|c|c|c|c|c|c|c|c|c|c|c|c|c|c|c|c|c|}
\hline \multirow[t]{3}{*}{ Social class } & \multirow{2}{*}{\multicolumn{6}{|c|}{ Live and stillbirths }} & \multicolumn{12}{|c|}{ Spontaneous abortions } & \multirow{2}{*}{\multicolumn{6}{|c|}{ Therapeutic abortions }} & \multirow[t]{3}{*}{ Total } \\
\hline & & & & & & & \multicolumn{6}{|c|}{ Examined } & \multicolumn{6}{|c|}{ Not examined } & & & & & & & \\
\hline & $L$ & $G$ & $\boldsymbol{B}$ & $M$ & $C$ & Total & $L$ & $G$ & $\boldsymbol{B}$ & $M$ & $C$ & Total & $L$ & $G$ & $\boldsymbol{B}$ & $M$ & $C$ & Total & $L$ & $\boldsymbol{G}$ & $\boldsymbol{B}$ & $M$ & $C$ & Tota & \\
\hline $\mathbf{I}+\mathbf{I I}$ & 5 & 23 & 19 & 4 & 1 & 52 & 1 & 0 & $4(1)$ & & 0 & $5(1)$ & 0 & 0 & 0 & $\mathbf{0}$ & 0 & 0 & 0 & $\mathbf{0}$ & 0 & $\mathbf{0}$ & 0 & 0 & $57(1)$ \\
\hline III N & 1 & 7 & 10 & 4 & 1 & 23 & 0 & $\mathbf{0}$ & 1 & 0 & $\mathbf{0}$ & 1 & 0 & 1 & 0 & $\mathbf{0}$ & 0 & 1 & $1(1)$ & 0 & 0 & $\mathbf{0}$ & 0 & $1(1)$ & $26(1)$ \\
\hline III M & 13 & 33 & 40 & 12 & 3 & 101 & 0 & 0 & 5 & 0 & 0 & 5 & 1 & 3 & 3 & 0 & 1 & 8 & 0 & $3(3)$ & $3(3)$ & 0 & $\mathbf{0}$ & $6(6) 1$ & $120(6)$ \\
\hline $1 V+V$ & 8 & 13 & 14 & 9 & 2 & 46 & 0 & 0 & 3 & 1 & 0 & 4 & 0 & 0 & 1 & 0 & 0 & 1 & 0 & 0 & 1(1) & $\mathbf{0}$ & 0 & $1(1)$ & $52(1)$ \\
\hline Unclassified & 1 & 2 & 7 & 1 & 2 & 13 & 0 & 0 & 0 & 0 & 0 & 0 & 0 & 0 & 0 & 0 & 0 & 0 & 0 & 0 & 0 & 0 & 0 & 0 & 13 \\
\hline Not known & 1 & 2 & 0 & 2 & 0 & 5 & 0 & 1 & 0 & 0 & 0 & 1 & 0 & 0 & 0 & 0 & 0 & 0 & 0 & 0 & 0 & $\mathbf{0}$ & 0 & 0 & 6 \\
\hline All & 29 & 80 & 90 & 32 & 9 & 240 & 1 & 1 & $13(1)$ & & 0 & $16(1)$ & 1 & 4 & 4 & 0 & 1 & 10 & $1(1)$ & $3(3)$ & $4(4)$ & 0 & 0 & $8(8)$ & $274(9)$ \\
\hline
\end{tabular}

Figures in parentheses are number of NTDs.

Table 9 Outcome of unsupplemented pregnancies in mothers with two previous NTD infants $(n=30$ : 1 twin pair) by centre and social class

\begin{tabular}{|c|c|c|c|c|c|c|c|c|c|c|c|c|c|c|c|c|c|c|c|c|}
\hline \multirow[t]{3}{*}{ Social class } & \multirow{2}{*}{\multicolumn{6}{|c|}{ Live and stillbirths }} & \multicolumn{7}{|c|}{ Spontaneous abortions } & \multirow{2}{*}{\multicolumn{6}{|c|}{ Therapeutic abortions }} & \multirow[t]{3}{*}{ Tota } \\
\hline & & & & & & & \multicolumn{6}{|c|}{ Examined } & \multirow{2}{*}{ Not examined } & & & & & & & \\
\hline & $L$ & $G$ & $\boldsymbol{B}$ & $M$ & $C$ & Total & $L$ & $G$ & $\boldsymbol{B}$ & $M$ & $C$ & Total & & $L$ & $G$ & $B$ & $M$ & $C$ & Total & \\
\hline $\begin{array}{l}\mathbf{I}+\mathbf{I I} \\
\text { III } \mathbf{N} \\
\text { III } \mathbf{M} \\
\text { IV }+ \text { V } \\
\text { Unclassified } \\
\text { All }\end{array}$ & $\begin{array}{l}0 \\
1 \\
2 \\
0 \\
0 \\
3\end{array}$ & $\begin{array}{l}0 \\
0 \\
4 \\
1 \\
0 \\
5\end{array}$ & $\begin{array}{l}2 \\
0 \\
7 \\
3(1) \\
2 \\
14(1)\end{array}$ & $\begin{array}{l}0 \\
0 \\
1 \\
1 \\
1 \\
3\end{array}$ & $\begin{array}{l}\mathbf{0} \\
\mathbf{0} \\
\mathbf{0} \\
\mathbf{0} \\
\mathbf{0} \\
\mathbf{0}\end{array}$ & $\begin{array}{l}2 \\
1 \\
14 \\
5(1) \\
3 \\
25(1)\end{array}$ & $\begin{array}{l}0 \\
0 \\
0 \\
0 \\
0 \\
0\end{array}$ & $\begin{array}{l}0 \\
0 \\
0 \\
0 \\
1 \\
1\end{array}$ & $\begin{array}{l}2 \\
0 \\
0 \\
0 \\
0 \\
2\end{array}$ & $\begin{array}{l}\mathbf{0} \\
\mathbf{0} \\
\mathbf{0} \\
\mathbf{0} \\
\mathbf{0} \\
\mathbf{0}\end{array}$ & $\begin{array}{l}\mathbf{0} \\
\mathbf{0} \\
\mathbf{0} \\
\mathbf{0} \\
\mathbf{0} \\
\mathbf{0}\end{array}$ & $\begin{array}{l}2 \\
0 \\
0 \\
0 \\
1 \\
3\end{array}$ & $\begin{array}{l}0 \\
0 \\
0 \\
0 \\
0 \\
0\end{array}$ & $\begin{array}{l}0 \\
0 \\
1(1) \\
0 \\
0 \\
1(1)\end{array}$ & $\begin{array}{l}0 \\
0 \\
2(2) \\
0 \\
0 \\
2(2)\end{array}$ & $\begin{array}{l}0 \\
0 \\
0 \\
0 \\
0 \\
0\end{array}$ & $\begin{array}{l}0 \\
0 \\
0 \\
0 \\
0 \\
0\end{array}$ & $\begin{array}{l}0 \\
0 \\
0 \\
0 \\
0 \\
0\end{array}$ & $\begin{array}{l}0 \\
0 \\
3(3) \\
0 \\
0 \\
3(3)\end{array}$ & $\begin{array}{c}4 \\
1 \\
17(3) \\
5(1) \\
4 \\
31(4)\end{array}$ \\
\hline
\end{tabular}

Figures in parentheses are number of NTDs. 


\section{Discussion}

In our preliminary paper we offered four possible interpretations of our data. No serious alternative has since arisen.

A group of women with a naturally low recurrence risk has unwittingly selected itself for supplementation. Some specific factors relating to recurrence risk deserve consideration.

\section{Social class}

Nevin ${ }^{11}$ has reported a social class gradient for NTD recurrence rates in Northern Ireland comparable with that for NTD incidence. Although there were fewer social class I + II mothers in the US group than in the FS, a significant $(P<0.02$, one tailed) difference in NTD recurrence favourable to vitamin supplementation remains if social classes III, IV, and V only are considered.

\section{Study centre}

The numbers of supplemented and unsupplemented mothers are similar for all centres except Belfast which has an excess of US mothers. As Northern Ireland is a high incidence area this might have weighted the US group with high recurrence risk mothers. In the event, recurrences in this group numbered $2(6 \%)$ of 35 in Leeds, $5(5 \%)$ of 95 at Guy's, and $6(5 \%)$ of 124 in Belfast. Furthermore, in our second cohort, still under study, most eligible mothers in Northern Ireland have been recruited for supplementation. Among those for whom the outcome of pregnancy or amniocentesis is known, the recurrence rate remains strikingly lower than in unsupplemented women.

\section{Outcome of previous pregnancy}

This was not considered in our first paper. Each FS mother was recruited when she was not pregnant and generally this was soon after the birth of the NTD child. By contrast, US mothers usually came to us when already pregnant and did not receive counselling. Consequently in the FS group a history of NTD in the immediately previous pregnancy is more common than in the US group $(79 \%$ compared with $60 \%$ ). The corollary is that spontaneous abortions ( $9 \%$ compared with $17 \%$ ) together with all other outcomes are under-represented in the FS group. Among those women in whom the previous pregnancy did not result in NTD, the proportions of immediately preceding pregnancies ending in spontaneous abortion are very similar, FS $17(42 \%)$ of 41 , US $48(40 \%)$ of 121 . There is known to be an increased frequency of spontaneous abortions before NTD births. It has been suggested that there is a causal connection, ${ }^{13}$ although this is disputed. ${ }^{14}$ It seems most unlikely that the deficit of earlier spontaneous abortions in our supplemented group could explain their low NTD recurrence rate.

Supplemented mothers aborted more NTD fetuses than did controls. The spontaneous abortion rates are comparable in FS, PS, and US mothers. Had US mothers entered the study before conception (as did FS mothers), their abortion rates might have been higher. Furthermore, of 32 spontaneous abortuses examined (FS 11, PS 2, US 19), only 1 had NTD. It seems highly improbable that the lower NTD recurrence rate in the offspring of supplemented mothers can be attributed to selective spontaneous abortions.

Something other than vitamin supplementation has reduced the incidence of NTDs in the treated group. It is common experience that any kind of intervention tends to influence other things (for example, diet surveys may alter eating habits). Furthermore, the tablets, in addition to their declared iron, calcium, and vitamin content, contain 13 other ingredients ( $R$ J Ancil, 1981, personal communication). The possibility that the effect is indirect could be tested only by a placebo study.

Vitamin supplementation has prevented some NTDs. This remains the most straightforward explanation and it is consistent with other published work in this field. It cannot however, be regarded as proved.

There are important implications for further research and, if the hypothesis is confirmed, for health care policies. An earlier proposal for a doubleblind placebo study was rejected by two research ethics committees, each for a different reason. These committees tend to record their decisions rather than their discussions and further retrospection seems pointless. However, the present position confirms the wisdom of our original intent because some important uncertainties remain. We regard placebo studies in high incidence areas as still desirable but ethically more difficult than before.

As regards the details of the supplementation regimen, the composition of the supplement and the duration of treatment deserve further study. Most of the earlier circumstantial evidence relates only to folic acid. Other studies ${ }^{4}$ also concern ascorbic acid and possibly riboflavin. It is desirable, and may be ethically acceptable, to try a more selective vitamin mix, or even folic acid alone. Regarding duration of treatment, data are accumulating from 'partially supplemented' mothers which may eventually answer this problem. In view of the practical 
difficulties of starting large-scale supplementation before conception it is important to continue studying mothers who begin supplements after conception. It is possible that a single dose taken on a specific day in the formation of the neural tube might be adequate $^{15}$ but this would be very difficult to achieve in humans. (Indeed, if the hypothesis that NTDs result from breakdown of a previously closed neural tube is correct, the deadline for possible benefit may be later.)

Turning to health care policies, the results of this study, if confirmed, could influence the cost/benefit ratio of serum AFP screening, and would stress the need to extend antenatal care into the preconceptional period.

We thank the patients who took part in this study and the doctors who referred them; Dr Jennifer Hanna and Dr Sheelagh Peel for follow up examinations; Mrs Jennifer Wild for data analysis; Miss Monica Heffernan, Miss Wendy Johnston, Mrs Claire Pownall, and Mrs Mary Weetman for home visits; Mrs Hilary Shaw for administrative and secretarial assistance.

This study was supported by Action Research for the Crippled Child, the Children's Research Fund, and Beecham Pharmaceuticals.

\section{References}

1 Hale F. Pigs born without eyeballs. $J$ Hered 1933; 24: 105-6.

2 Warkany J, Nelson R C. Skeletal abnormalities in the offspring of rats reared on deficient diets. Anat Rec 1941; 79: $83-100$.

3 Smithells R W, Ankers C, Carver M E, Lennon D, Schorah C J, Sheppard S. Maternal nutrition in early pregnancy. Br J Nutr 1977; 38: 497-506.
4 Smithells R W. Sheppard S, Schorah C J. Vitamin deficiencies and neural tube defects. Arch Dis Child 1976; 51: 944-50.

5 Hibbard E D, Smithells R W. Folic acid metabolism and human embryopathy. Lancet 1965; i: 1254.

6 Pritchard J A, Scott D E, Whalley P J, Haling R F, Jr. Infants of mothers with megaloblastic anemia due to folate deficiency. J Am Med Soc 1970; 211 : 1982-4.

7 Laurence K M, James N, Miller M, Campbell $\mathbf{H}$. Increased risk of recurrence of pregnancies complicated by fetal neural tube defects in mothers receiving poor diets, and possible benefit of dietary counselling. $\mathrm{Br} \mathrm{Med}$ $J$ 1980; 281 : 1592-4.

8 Smithells R W, Sheppard S, Schorah C J, et al. Possible prevention of neural tube defects by periconceptional vitamin supplementation. Lancet 1980; i: 339-40.

9 Wynn V. Vitamins and oral contraceptive use. Lancet 1975; i: 561-4.

10 Gal I, Sharman I M, Pryse-Davies J. Vitamin A in relation to human congenital malformations. $A d v$ Teratol 1972; 5: 143-59.

11 Nevin N C. Letter: Recurrence risk of neural tube defects. Lancet 1980; i: 1301-2.

12 Record R G, McKeown T. Congenital malformations of the central nervous system. II. Maternal reproductive history and familial incidence. $\mathrm{Br} J$ Soc Med 1950; 4: 26-50.

13 Clarke C A, Hobson D, McKendrick O M, Rogers S C, Sheppard P M. Spina bifida and anencephaly: miscarriage as possible cause. $\mathrm{Br}$ Med J 1975; iv: 743-6.

14 James W H. Birth ranks of spontaneous abortions in sibships of children affected by anencephaly or spina bifida. Br Med J 1978; i: 72-3.

15 Seller M J, Embury S, Polani P E, Adinolfi M. Neural tube defects in curly-tail mice. II. Effect of maternal administration of vitamin A. Proc $R$ Soc Lond [Biol] 1979; 206: 95-107.

Correspondence to Professor R W Smithells, Department of Paediatrics and Child Health, University of Leeds, 27 Blundell Street, Leeds LS1 3ET.

Received 19 March 1981 